\title{
Three-Stage Serially Concatenated Codes and Iterative Center-Shifting $K$-Best Sphere Detection for SDM-OFDM: An EXIT Chart Aided Perspective
}

\author{
Li Wang, Lei Xu, Sheng Chen and Lajos Hanzo \\ School of ECS, University of Southampton, SO17 1BJ, UK. \\ Tel: +44-23-8059 3125, Fax: +44-23-8059 4508 \\ Email: $\{1 w 05 r, 1 x 04 r$, sqc, lh\}@ecs.soton.ac.uk; http://www-mobile.ecs.soton.ac.uk
}

\begin{abstract}
Iterative $K$-best sphere detection (SD) and channel decoding is appealing, since it is capable of achieving a nearmaximum-a-posteriori (MAP) performance at a low complexity. However, a potentially excessive computational cost is imposed, especially in a high-throughput Spatial Division Multiplexing (SDM) aided OFDM system emloying a large number of transmit antennas and/or high-order modulation schemes. This problem is further aggravated, when the number of transmit antennas exceeds that of the receive antennas, namely in the challanging scenario of rank-deficient systems. In order to further reduce the complexity imposed, we propose a unity-rate-code-aided (URC) three-stage concantenated transceiver constituted by an inner and outer channel encoder/decoder pair as well as the SDM aided OFDM transmitter and $K$-best SD. We have demonstrated that our proposed three-stage scheme is capable of achieving a substantial performance gain over the classic two-stage scheme. For example, given a target Bit Error Ratio (BER) of $10^{-5}$, the three-stage SD-aided receiver is capable of achieving a performance gain of $4 \mathrm{~dB}$ over its two-stage counterpart in an $(8 \times 4)$-element SDM/OFDM system. Furthermore, we have also investigated the achievable performance of our novel centershifting aided SD in the context of this three-stage scheme. Consequently, an additional $0.5 \mathrm{~dB}$ performance gain can be attained. Finally, the convergence behavior of the proposed schemes were studied with the aid of 3D EXtrinsic Information Transfer (EXIT) charts and their 2D projections.
\end{abstract}

\section{Motivation}

Multiple-input-multiple-output (MIMO) systems exhibit a high throughput potential, since their capacity may be shown to increase linearly with the channel, SNR, as apposed to the logorithmic improvements imposed by the Shannon-Hartley law. However, in order to achieve the optimum BER performance in a MIMO-aided system, the classic maximum likelihood (ML) detector conducts an exhaustive search over the entire $\mathcal{M}_{c}{ }^{M}$-element search space of the $M$-transmitter MIMO scheme using $\mathcal{M}_{c}$-ary modulation. Hence, the ML detector exhibits a potentially excessive computational complexity. It was shown in [1-3] that sphere detection (SD) is capable of attaining a near-ML performance at a significantly reduced complexity. Motivated by the original SD proposals of [1-3], diverse further complexity reduction schemes have been proposed for example in $[2,4,5]$. Nonetheless their complexity may still become potentially unaffordable in highthroughput systems invoking high-order modulation schemes and/or a high number of transmit antennas. This complexity problem may be further aggravated in rank-deficient systems, where the number of transmit antennas exceeds that of the receiver antennas. The novelty of this paper is that we further reduced the SD's complexity upon combining unit-rate-code
(URC) having an Infinite Impulse Response (IIR) and its corresponding decoder with the channel-coded SDM-OFDM transmitter and the receiver, respectively, which results in a three-stage serially concatenated scheme. This three-stage scheme was shown to result in a significant performance gain in the context of the single-input single-output MMSE turbo equlizer of [6], which motivates its employment in the SDM/SDMA MIMO-OFDM context of this paper.

The rest of this paper is organised as follows. Section II describes the system model of our MIMO system. The proposed $K$-best SD aided three-stage transceiver is described in Section III, where an EXIT chart based analysis is conducted. A novel SD technique and its performance attained in the context of the proposed three-stage scheme are characterized in Section IV. Finally, in Section V we offer our conclusions.

\section{SySTEM MODEL}

Consider the following generic model constructed for MIMO systems employing $M$ number of transmit antennas and $N$ number of receive antennas [7]:

$$
\mathbf{y}=\mathbf{H s}+\mathbf{w},
$$

where $\mathbf{y} \in \mathbb{C}^{N \times 1}, \mathbf{s} \in \mathbb{C}^{M \times 1}$ and $\mathbf{w} \in \mathbb{C}^{N \times 1}$ denote the received signal and transmitted signal vector as well as the AWGN sample vector, respectively. Moreover, the frequencydomain channel transfer factor (FDCHTF) matrix $\mathbf{H}$ is a $(N \times M)$-dimensional independent and identically distributed (i.i.d.) zero-mean unit variance complex Gaussian matrix, known perfectly to the receiver, with each column representing the unique spatial signature of the corresponding transmit antenna [7]. Furthermore, the AWGN noise, $w_{n}$ encountered at the $n$th receive antenna element exhibit a zero-mean and a variance of $\sigma_{w}^{2}$.

\section{Sphere-Detection-Aided Three-Stage ITERATIVE RECEIVER}

\section{A. K-Best List Sphere Detection}

With the aid of the unconstrained ML solution of $\hat{\mathbf{x}}_{c}=$ $\left(\mathbf{H}^{H} \mathbf{H}+\sigma_{w}^{2} \mathbf{I}\right) \mathbf{H}^{H} \mathbf{y}$ and the Cholesky factorization of the channel's FDCHTF [1], the well-known ML solution may be formulated as [7]:

$$
\hat{\mathbf{s}}_{M L}=\arg \min _{\check{\mathbf{s}} \in M_{c}^{M}}\|\mathbf{y}-\mathbf{H} \check{\mathbf{s}}\|_{2}^{2},
$$

where again, $M_{c}$ is the number of modulated symbol points in the constellation and $M$ is the number of transmit antennas 

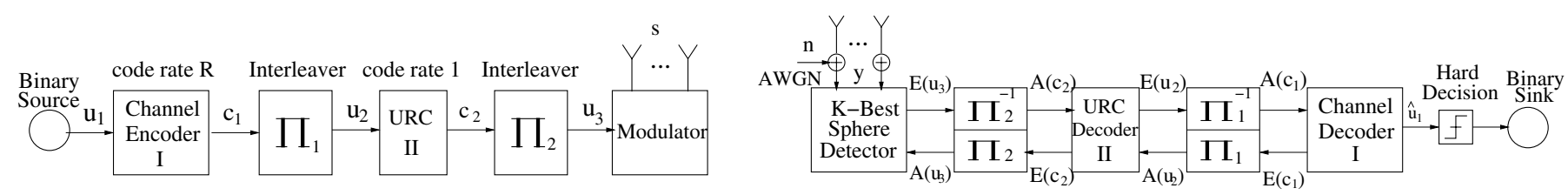

Fig. 1. Unit-Rate-Code-Aided 3-Stage Iterative Detection Scheme

employed by the system. The ML solution of Eq.(2) may be transformed into [8]:

$$
\begin{gathered}
\hat{\mathbf{s}}_{M L}=\arg \min _{\check{\mathbf{s}} \in M_{c}^{M}}\left(\check{\mathbf{s}}-\hat{\mathbf{x}}_{c}\right)^{H} \mathbf{U}^{H} \mathbf{U}\left(\check{\mathbf{s}}-\hat{\mathbf{x}}_{c}\right) \\
=\arg \min _{\check{\mathbf{s}} \in M_{c}^{M}} \sum_{i=1}^{M} \underbrace{u_{i i}^{2}\left[\check{s}_{i}-\hat{x}_{i}+\sum_{j=i+1}^{M} \frac{u_{i j}}{u_{i i}}\left(\check{s}_{j}-\hat{x}_{j}\right)\right]^{2},(4)}_{\phi}
\end{gathered}
$$

where $\mathbf{U}$ is a $(M \times M)$-element upper-triangular matrix, which satisfies $\mathbf{U}^{H} \mathbf{U}=\mathbf{H}^{H} \mathbf{H}+\sigma_{w}^{2} \mathbf{I}_{\mathcal{C}}$. For the $K$-best SD [3], instead of considering all legitimate bit combinations at each tree search level, we only retain a fixed number of $K$ decision states also referred to as decision nodes, namely those that have the smallest accumulated Partial Euclidean Distances (PEDs) from the SD's initial search center, constituted for example by the classic MMSE solution [7], where the PEDs correspond to the term $\phi$ of Eq.(4). The corresponding searchtree was exemplified in [9]. Hence, after the search reaches the tree leaf level, a candidate list $\mathcal{L}$ is generated, which contains $N_{\text {cand }}=K$ number of $\left(\mathcal{M}_{c} \cdot M\right)$-bit candidate solutions, which are then used for the extrinsic Log-LikelihooldRatio (LLR) calculation by the iterative Soft-Input-Soft-Output (SISO) receiver [2].

\section{B. Sphere-Detection-Aided Three-Stage Iterative Receiver}

Figure 1 depicts the system model of the SD-aided 3-stage serially concatenated transceiver in the context of a multipleantenna-aided SDM/OFDM system. At the transmitter, a block of $L$ information bits $u_{1}$ is first encoded by the convolutional channel encoder I in order to generate the coded bits $c_{1}$, which are interleaved by the interleaver $\Pi_{1}$ of Figure 1 . Then the resultant permuted bits $u_{2}$ are fed through the URC encoder II and the interleaver $\Pi_{2}$, yielding the interleaved doubleencoded bits $u_{3}$, which are delivered to the bit-to-symbol modulator/mapper. Note that the labels $u$ and $c$ represent the uncoded and coded bits, respectively, corresponding to the specific module indicated by the subscript. For example, $u_{2}$ and $c_{2}$ denote the uncoded bits and the coded bits at the input and the output of the URC encoder II of Figure 1, respectively. At the receiver of Figure 1, which is constituted by three modules, namely the SD, the URC decoder II and the convolutional channel decoder II, the extrinsic information is exchanged amongst the blocks in a number of consecutive iterations. Specifically, as shown in Figure $1, A(\cdot)$ represents the a priori information expressed in terms of the LLRs, while $E(\cdot)$ denotes the corresponding extrinsic information. Hence, the URC decoder generates two extrinsic outputs by processing two a priori inputs delivered from both the SD and the convolutional decoder II. After completing the last iteration, the estimates $\hat{u}_{1}$ of the original transmitted information bit $u_{1}$ are produced by the convolutional channel decoder I.

\section{3D EXIT Chart Analysis}

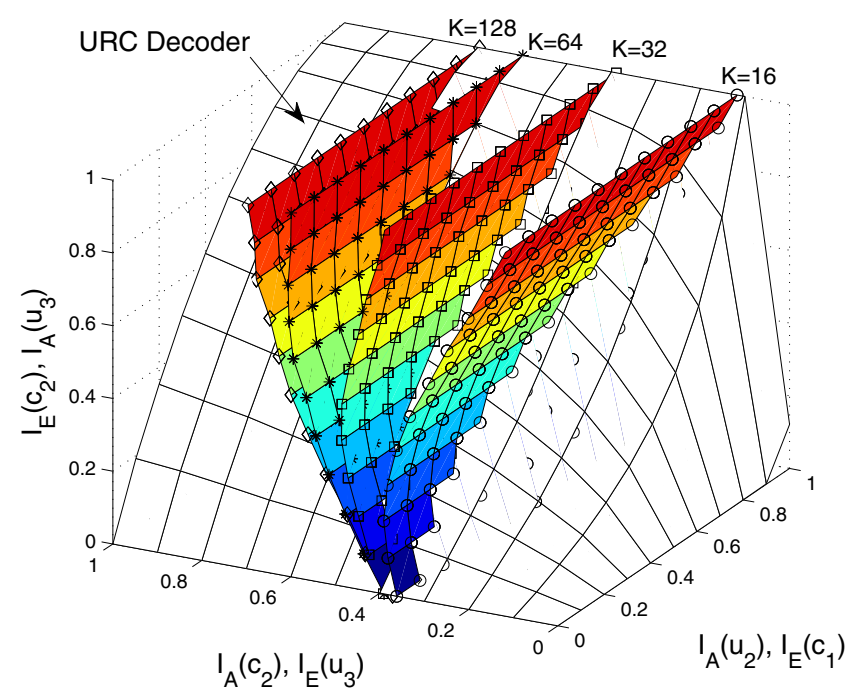

(a)

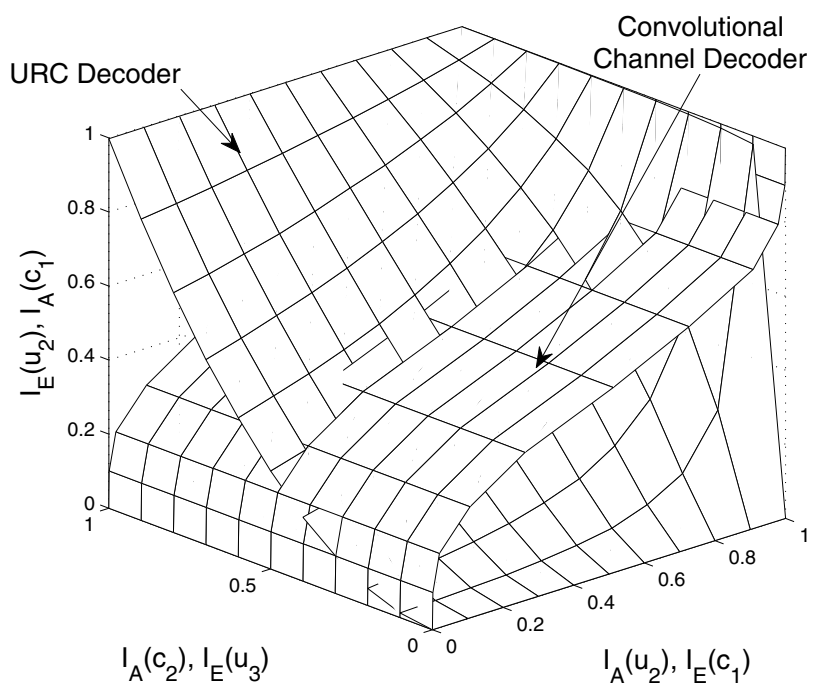

(b)

Fig. 2. $\quad(\mathrm{SNR}=8 \mathrm{~dB}) 3 \mathrm{D}$-EXIT Charts of K-Best SD-Aided 3-Stage Iterative Receiver In the Scenario of $(8 \times 4)$ Rank-Deficient 4-QAM SDMA/OFDM System.

We denote the mutual information (MI) between the a priori value $A(b)$ and the bit $b$ as $I_{A(b)}$, while the MI between the extrinsic value $E(b)$ and the bit $b$ by $I_{E(b)}$. Hence, the MI 
of the two outputs of the URC decoder, namely, $I_{E\left(u_{2}\right)}$ and $I_{E\left(c_{2}\right)}$, are functions of the two a priori MI inputs, namely, $I_{A\left(u_{2}\right)}$ and $I_{A\left(c_{2}\right)}$. Explicitly, we have [6]:

$$
\begin{aligned}
& I_{E\left(u_{2}\right)}=T_{u_{2}}\left(I_{A\left(u_{2}\right)}, I_{A\left(c_{2}\right)}\right), \\
& I_{E\left(c_{2}\right)}=T_{c_{2}}\left(I_{A\left(u_{2}\right)}, I_{A\left(c_{2}\right)}\right) .
\end{aligned}
$$

Therefore, two 3D EXIT charts corresponding to the above two equations are needed in order to fully describe the EXIT characteristics of the URC decoder, as portrayed in Figures 2(a) and 2(b), respectively. In contrast to the double-inputdouble-output URC module of Figure 1, both the SD and the convolutional decoder can be viewed as single-input-singleoutput modules, for a given received signal vector. Thus, a single 2D EXIT chart is sufficient for characterizing each of them. Similarly, we have the corresponding EXIT functions expressed as [6]:

$$
I_{E\left(u_{3}\right)}=T_{u_{3}}\left(I_{A\left(u_{3}\right)}, E_{b} / N_{0}\right)
$$

for the SD and

$$
I_{E\left(c_{1}\right)}=T_{c_{1}}\left(I_{A\left(c_{1}\right)}\right)
$$

for the convolutional channel decoder. We note that since the MI $I_{E\left(u_{3}\right)}$ of the SD's output is independent of $I_{A\left(u_{2}\right)}$, the traditional EXIT curve of the SD portrayed in the 2D space can be extended to the 3D space by sliding the EXIT curve along the $I_{A\left(u_{2}\right)}$ axis as depicted in Figure 2(a), where four EXIT surfaces of the $K$-best SD corresponding to four different values of $K$ are plotted. Similarly, the EXIT surface of the outer convolutional decoder can be generated, as depicted in Figure 2(b) together with the other 3D EXIT chart of the URC decoder, since $I_{E(c 1)}$ of Eq.(8) is independent of $I_{A(c 2)}$. Consequently, two 3D EXIT charts are required for plotting all the EXIT functions. To be specific, Figure 2(a) was generated from Eq.(6) and Eq.(7), while Figure 2(b) from Eq.(5) and Eq.(8).

\section{2D EXIT Chart Analysis and Simulation Results}

The intersection of the surfaces of the SD and the URC decoder characterizes the best possible achieveable performance for different fixed values of $I_{A\left(u_{2}\right)}$, as the iterations between the SD and the URC decoder are carried out by exchanging their soft bit information. More importantly, according to Eq.(5), for each point $\left[I_{A\left(u_{2}\right)}, I_{A\left(c_{2}\right)}, I_{E\left(c_{2}\right)}\right]$ of the intersection line seen in Figure 2(a), there is a specific point $\left[I_{A\left(u_{2}\right)}, I_{A\left(c_{2}\right)}, I_{E\left(u_{2}\right)}\right]$ determined by the two a priori inputs of the URC decoder on the surface of the URC decoder in Figure 2(b). Hence, there must be a line (not plotted) on the surface of the URC decoder in Figure 2(b) corresponding to the line of intersection in Figure 2(a). In order to simplify the complicated 3D EXIT chart representation, we view the $\mathrm{SD}$ and the URC decoder as a joint module, having the single input $I_{E\left(u_{2}\right)}$ and the single output $I_{A\left(u_{2}\right)}$. As a result, a classic 2D EXIT chart can be plotted, which can be also obtained by projecting the aforementioned line on the surface of the URC decoder seen in Figure 2(b) on the $I_{E\left(u_{2}\right)}-I_{A\left(u_{2}\right)}$ plane, as seen in Figure 3(a).

Figure 3(a) shows the 2D EXIT chart of decoder I and the combined module constituted by decoder II and the SD, in comparison to that of the conventional two-stage iterative

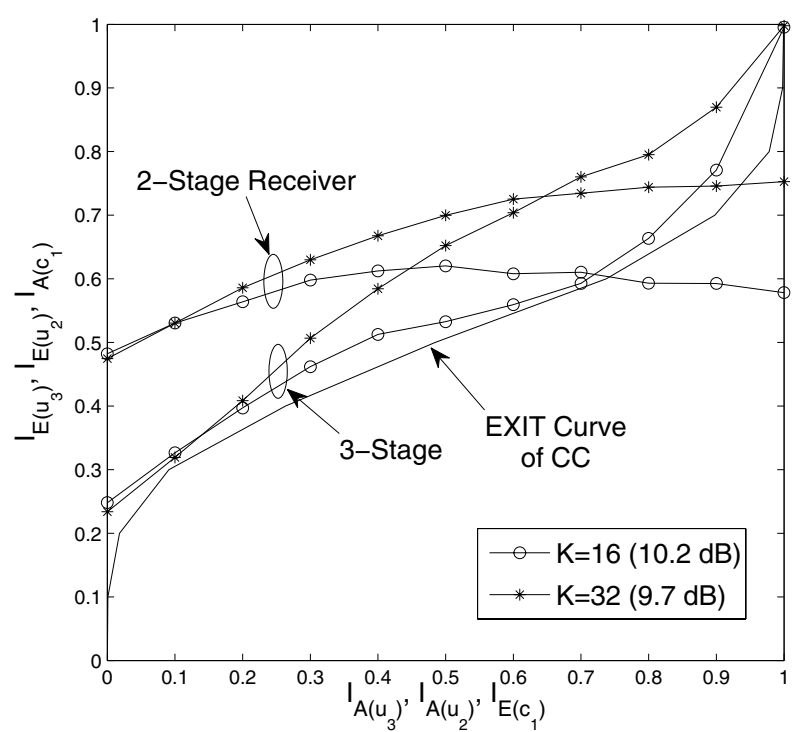

(a) 2D EXIT Chart

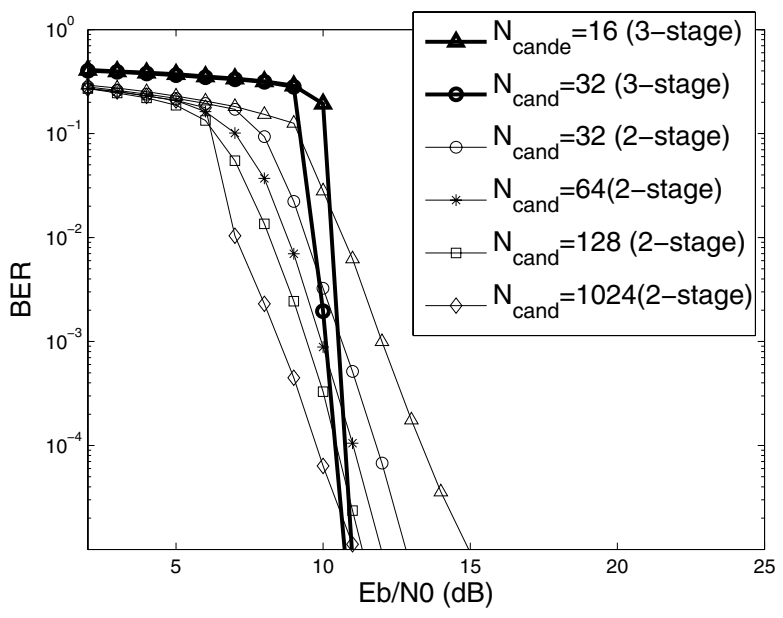

(b) BER Performance

Fig. 3. EXIT analysis and BER performance of the three-stage iterative receiver using the $K$-best $\mathrm{SD}$.

receiver. As observed in Figure 3(a), due to the insufficiently high length of the candidate list, the maximum achievable iterative gain of the traditional two-stage receiver becomes rather limited, since the EXIT curve of the SD intersects with that of the channel decoder at an earlier stage, if we have $K$ or $N_{\text {cand }}$ values of 16 or 32 . In other words, when the resultant decoding trajectory gets trapped at the intersection point of the EXIT chart, where the decoding convergence is curtailed after a certain number of iterations, typically a residual error floor persists. However, with the aid of the URC decoder II seen in Figure 1, the point of EXIT curve intersection of the joint decoder II and SD module and that of decoder I moves closer to the $(1,1)$ point, resulting in a near-error-free performance, as long as there is an open tunnel between the two EXIT curves. More specifically, as observed in Figure 3(a), for the SD-aided iterative receiver using $K=16$, an open tunnel is created between the EXIT curve of the joint SD 


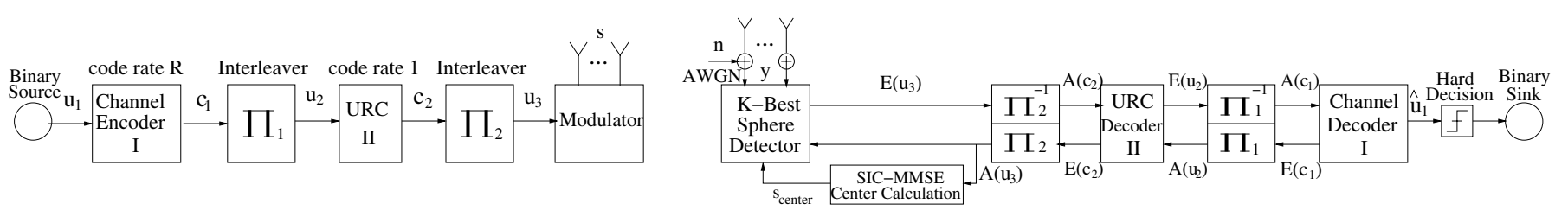

Fig. 4. Receiver Architecture of the SIC-MMSE-aided iterative center-shifting $K$-best SD scheme.

and decoder II module and that of decoder I at an SNR of $10.2 \mathrm{~dB}$. Accordingly, the corresponding BER curve plotted in Figure 3(b) confirmed the predictions of the EXIT chart analysis seen in Figure 3(a), indicating that the BER decreases sharply, once the SNR is in excess of about $10.2 \mathrm{~dB}$. Similarly, when we have $K=32$, experiencing a lower convergence threshold of $9.7 \mathrm{~dB}$ results in an even earlier reduction of the BER, as shown in Figure 3(b). Consequently, given a target BER of $10^{-5}$, a nearly $4 \mathrm{~dB}$ performance gain can be attained by the 3 -stage scheme over the conventional 2 -stage iterative receiver, when employing the URC decoder II and $K=16$. When comparing the 3-stage and 2-stage arrangements for $K=32$, the former had a $2 \mathrm{~dB}$ gain. However, as a price, the BER of the three-stage scheme is expected to be higher than that of the 2-stage receiver at low SNRs. The reason behind this phenomenon becomes clearer, if we refer to the EXIT chart comparison of the 2-stage and 3-stage iterative receivers characterized in Figure 3(a), where we observe that the EXIT curve of the SD of the conventional 2-stage receiver has a significantly higher starting point than that of its 3 -stage counterpart, resulting in a lower $E_{b} / N_{o}$ convergence threshold, which in turn leads to a potentially higher iterative gain at relatively low SNRs. Although the employment of the URC encoder/decoder pair at the transmitter/receiver is capable of moving the EXIT curve intercept point closer to $(1,1)$, an open tunnel can only be formed, if the value of $K$ or $N_{\text {cand }}$ as well as that of the SNR is sufficiently high. This explains, why the BER curve of the SD using $K=32$ drops sharply at a lower SNR than that of the SD employing $K=16$, as seen in Figure 3(b).

\section{Center-Shifting-Sphere-Detection-Aided THREE-STAGE RECEIVER}

\section{A. MMSE Soft-Interference-Cancellation Aided Center-} Shifting Technique for SD

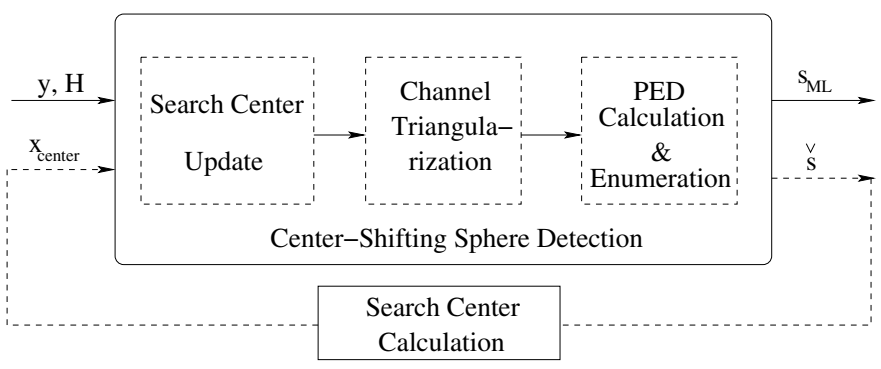

Fig. 5. The structure of the iterative SD using center-shifting scheme.

According to Eq.(4), when using sphere detection, the ML solution can be found by creating a reduced-size search-hypersphere centered around the MMSE solution by choosing an appropriate value for $K$. During our investigations, we realized that it would be desirable to set the SD's search center to a MIMO signal constellation point, which may be expected to be closer to the real ML solution than the MMSE solution, because this would allow us to reduce the SD's search space and hence its complexity. To some extent, extending the SD's search from a more accurate search center can be considered as a process of search-complexity reduction. As a result, the affordable computational complexity can be flexibly split between the center calculation phase and the search phase of Figure 5, where the triangularization of the channel matrix $\mathbf{H}$ and the PED calculation previously detailed in Section III-A is portrayed explicitly. For more details about the center-shifting scheme, please refer to [10], where we employed the MMSE soft-interference-cancellation (SIC-MMSE) algorithm [11] to calculate the search center based on the a priori information provided by the channel decoder.

\section{B. SIC-MMSE-Aided Iterative Center-Shifting SD Assisted Three-Stage Receiver Architecture}

Based on the above discussions, we propose the SICMMSE center-shifting SD aided three-stage receiver portrayed in Figure 4, where re-detection using an updated search center generated by the SIC-MMSE algorithm has to be carried out during each iteration that invokes the SD. The benefits of applying the SIC-MMSE center-shifting scheme become more explicit, if we refer to the resultant 3D EXIT surfaces of the SD in conjunction with different values of $K$, as depicted in Figure 6. Specifically, in contrast to the EXIT surface of the SD dispensing with center-shifting in Figure 2(a), the EXIT surface of the center-shifting-aided SD seen in Figure 6 does not suffer to the same extent from having an EXITchart constriction even if $K$ is set to a small value, e.g. 16 . Hence, the resultant projected EXIT curve of the combined SD and URC decoder II module plotted in Figure 7(a) for $K=N_{\text {cand }}=16$ exhibits a potentially wider open tunnel. To be more specific, the original convergence threshold of the three-stage receiver using no center-shifting is about 10.2 $\mathrm{dB}$, since an open tunnel is just formed for SNRs in excess of this level. For SNRs below this level, the EXIT curve of the combined module would fall below that of decoder I, leading to a consistently closed EXIT tunnel, which is exemplified by the situation characterized by $\mathrm{SNR}=9.6 \mathrm{~dB}$, as also portrayed in Figure 7(a). However, with the aid of the SIC-MMSE-aided center-shifting scheme, a wider open tunnel has been created between the EXIT curves. The convergence threshold of the SIC-MMSE center-shifting aided three-stage scheme was reduced to about $9.6 \mathrm{~dB}$ and the resultant BER curve is plotted in Figure 7(b) in comparison to that of the three-stage receiver dispensing with center-shifting. Indeed, with the aid of the center-shifting scheme, the BER curve starts to drop more sharply at a slightly lower SNR, which is 
similar to the convergence threshold observed in Figure 7(a), yielding a performance gain of $0.5 \mathrm{~dB}$ at the target BER of $10^{-5}$. It is not unexpected that the attainable performance improvement is insignificant, since the SIC-MMSE centershifting scheme fails to increase the relatively low starting point at zero abscissa value of the EXIT curve, which is determined by the employment of the URC decoder II.

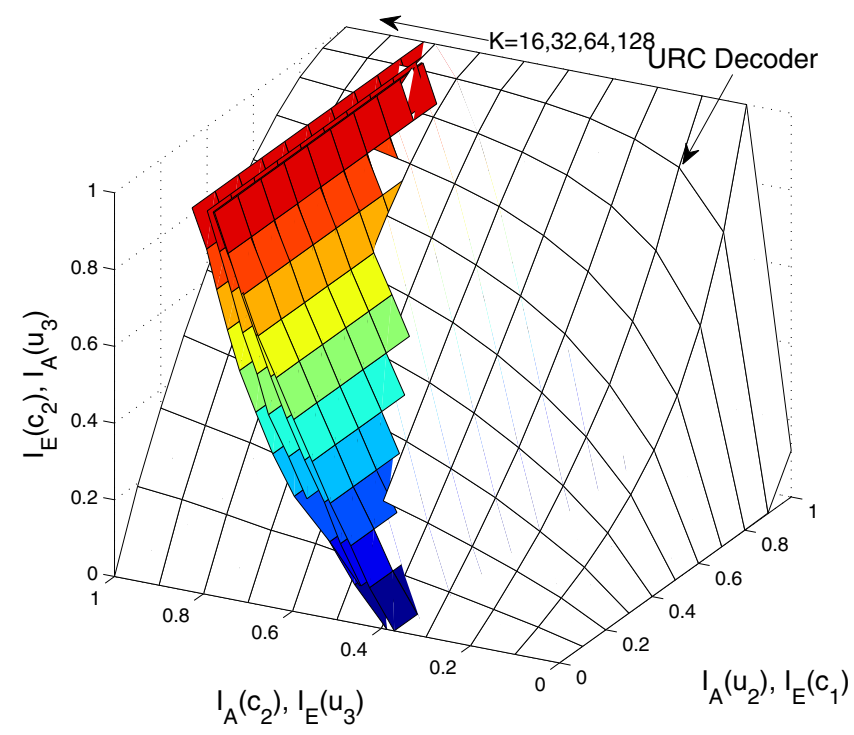

Fig. 6. 3D EXIT chart of the three-stage SIC-MMSE-aided center-shifting $K$-best SD assisted iterative receiver

\section{CONCLUSION}

A sphere detection aided three-stage scheme was proposed, which is capable of achieving a substantial BER performance gain in comparison to the classic two-stage scheme, provided that the SNR is sufficiently high. For example, given a target BER of $10^{-5}$, the three-stage SD-aided receiver is capable of achieving an SNR gain of $4 \mathrm{~dB}$ over its two-stage counterpart in an $(8 \times 4)$-element SDM/OFDM system. Furthermore, an additional $0.5 \mathrm{~dB}$ SNR gain can be attained with the aid of the novel center-shifting based SD advocated.

\section{ACKNOWLEDGEMENTS}

The authors would like to thank Dr. S. X. Ng for the discussions on the EXIT characteristics of the proposed system.

\section{REFERENCES}

[1] E. Viterbo and J. Boutros, "A universal lattice code decoder for fading channels," IEEE Transactions on Information Theory, vol. 45, pp. 16391642, July 1999.

[2] B. M. Hochwald and S. ten Brink, "Achieving near-capacity on a multiple-antenna channel," IEEE Transactions on Communications, vol. 51, pp. 389-399, Mar. 2003.

[3] K. Wong, C. Tsui, R. S. K. Cheng, and W. Mow, "A VLSI architecture of a k-best lattice decoding algorithm for MIMO channels," Proc. IEEE International Symposium on Circuits and Systems, vol. 3, pp. 273-276, May 2002.

[4] A. M. Chan and I. Lee, "A new reduced-complexity sphere decoder for multiple antenna systems," Proc. ICC, vol. 1, pp. 460-464, Apr./May 2002.

[5] A. Wolfgang, J. Akhtman, S. Chen, and L. Hanzo, "Reducedcomplexity near-maximum-likelihood detection for decision feedback assisted space-time equalization," IEEE Transactions on Wireless Communications, vol. 3, pp. 2407 - 2411, 2007.

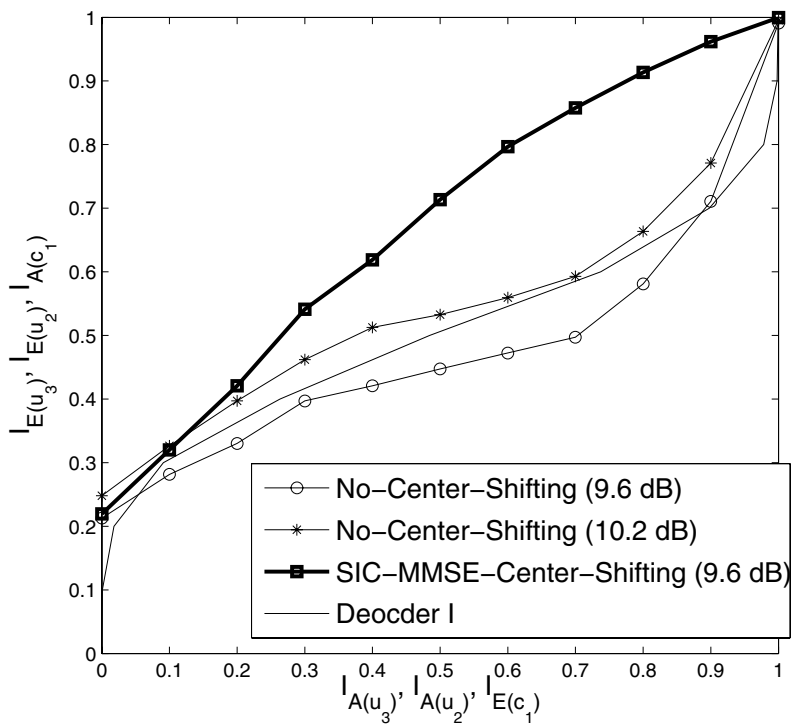

(a) 2D EXIT Chart $(K=16)$

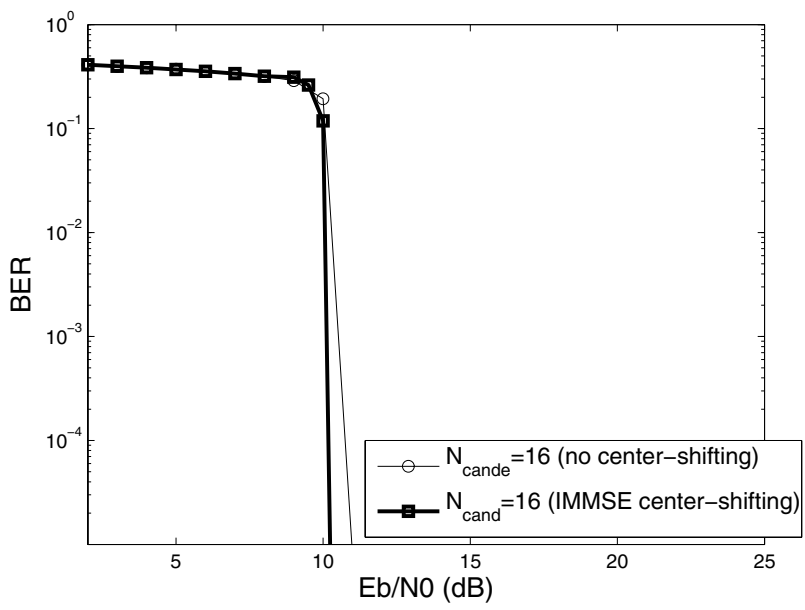

(b) BER Performance

Fig. 7. EXIT Analysis and BER Performance of SIC-MMSE-Aided CenterShifting K-Best SD 3-Stage Iterative Receiver.

[6] J. Wang, S. X. Ng, L. L. Yang, and L. Hanzo, "Combined serially concatenated codes and MMSE equalization: An EXIT chart aided perspective," Proc. VTC-2006 Fall,, pp. 1-5, Sept. 2006.

[7] L. Hanzo, M. Munster, B. J. Choi, and T. Keller, OFDM and MC-CDMA for Broadband Multi-User Communications, WLANs and Broadcasting. IEEE Press, 2003

[8] T. Cui and C. Tellambura, "An efficient generalized sphere decoder for rank-deficient MIMO systems," Proc. VTC2004-Fall,, vol. 5, pp. 36893693, Sept. 2004.

[9] L. Hanzo and T. Keller, OFDM and MC-CDMA : a primer. John Wiley, 2006.

[10] L. Wang, L. Xu, S. Chen, and L. Hanzo, "MMSE soft-interferencecancellation aided iterative center-shifting $K$-best sphere detection for MIMO channels," http://eprints.ecs.soton.ac.uk/14523.

[11] M. Tuchler, A. C. Singer, and R. Koetter, "Minimum mean squared error equalization using a priori information," IEEE Transactions on Signal Processing, vol. 50, pp. 673-683, Mar. 2002. 